Full Length Article

\title{
Practical prognostic score for predicting the extent of resection and neurological outcome of gliomas in the sensorimotor area
}

\author{
Giannantonio Spena $^{\mathrm{a}, *, 1}$, Federico D’Agata ${ }^{\mathrm{b}, 1}$, Pier Paolo Panciani ${ }^{\mathrm{a}, 1}$, Luciano Buttolo ${ }^{\mathrm{a}, 1}$, \\ Michela Buglione di Monale Bastia ${ }^{\mathrm{c}, 1}$, Marco Maria Fontanella ${ }^{\mathrm{a}, 1}$

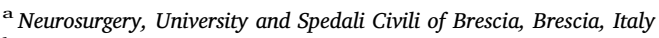 \\ ${ }^{\mathrm{b}}$ Neuroscience, University of Turin, Turin, Italy \\ ${ }^{\mathrm{c}}$ Radiotherapy, University and Spedali Civili of Brescia, Brescia, Italy
}

\section{A R T I C L E I N F O}

\section{Keywords:}

Gliomas

Monitoring

Functional

Score

Outcome

Awake surgery

Craniotomy

\begin{abstract}
A B S T R A C T
Objective: In this prospective study, we assessed the utility of a novel prognostic score (PS) in guiding the surgical strategy of patients with sensorimotor area gliomas.

Patients and methods: Form December 2012 to April 2016, we collected data from patients diagnosed with brain gliomas in the sensorimotor area. All the patients had intraoperatively confirmed contiguity or continuity with sensorimotor cortical and subcortical structures. Several clinical and radiological factors were analyzed to generate a PS for each patient (range 1-8). The end-points included the extent of resection (EOR) and neurological outcome (modified Rankin Score; mRS). We assessed the predictive power of the PS using different analyses. Crosstabs analyses and Fisher's exact test (Fet) were used to evaluate the possible predictive parameters, and for the classification of positive or negative outcomes for the chosen proxies; the significance threshold was set at $\mathrm{p}<0.05$.

Results: Using independent $t$-tests, we compared the mRS at different time points (pre, post, and at 6 months) for 2 subgroups from the total sample using a cut-off PS value of 4 . For the EOR, a PS value of $\geq 5$ was predictive of successful outcome, a value of 4 indicated an uncertain outcome, and a value of $\leq 3$ predicted a worse outcome. Conclusions: This PS value can be easily used in clinical settings to help predict the functional outcome and EOR in sensorimotor area tumors. Integration with information from fMRI, DTI, and TMS, along with MRI spectroscopy could further enhance the value of this PS.
\end{abstract}

\section{Introduction}

The surgical approach for gliomas in highly functional areas aims to achieve large surgical resection to improve oncological prognosis and functional preservation to maintain an optimal postoperative functional status [1]. This objective of wider resection is easily achievable in certain locations, but may be difficult at certain other sites, including an eloquent area or more generally critical area. Furthermore, an oncologically desirable resection always should be balanced with the need to preserve neurological function, in both high and low grade gliomas (HGGs and LGGs). Data from recent studies suggest that poor postoperative functional outcome negatively affects the quality of adjuvant therapies, and finally the global outcome [2-4]. Although clear evidence has been obtained regarding the factors predicting survival (age, tumor volume, preoperative neurological status, and location in eloquent areas) [5-13], information on reliable indices predicting functional outcome and EOR is scarce. Moreover, an eloquent location can hinder larger resection and is associated with a greater risk of postoperative deficits. Nevertheless, the availability of the direct mapping technique has helped overcome this limitation, and facilitates safe and large resection by exploiting interindividual variability and brain plasticity [14-18]. However, it would be useful to determine the specific patient characteristics that enhance the risk of new neurological deficits. In fact, the ultimate goal involves the ability to predict the risk of a specific neurologic deficit for a given lesion at a particular site in the brain [19]. In our previous retrospective study [20], we assessed the gliomas in eloquent areas to determine the clinical and neuroradiological parameters that predominantly affect the extent of resection (EOR) and immediate and late neurological outcome. We observed that factors related to the biology and morphology of the tumor, along with the clinical presentation, clearly helped define the operative risks in terms of the EOR and functional outcome. Based on this evidence, we

\footnotetext{
* Corresponding author at: Piazzale Spedali Civili 1, 25121 Brescia, Italy.

E-mail address: giannantonios@hotmail.com (G. Spena).

${ }^{1}$ These authors contributed equally to this work.
} 


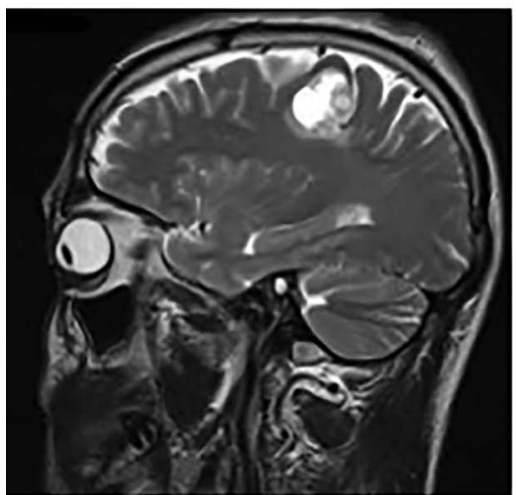

class 1: tumors invading and confined to only 1 gyrus without infiltration of white matter connections
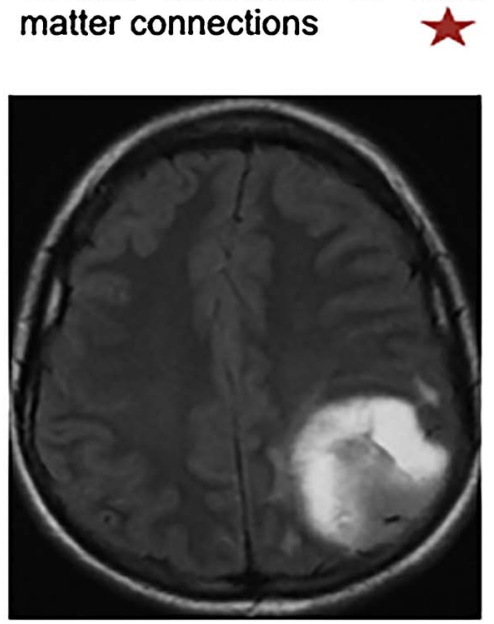

the same as class 3 but with a large cystic component

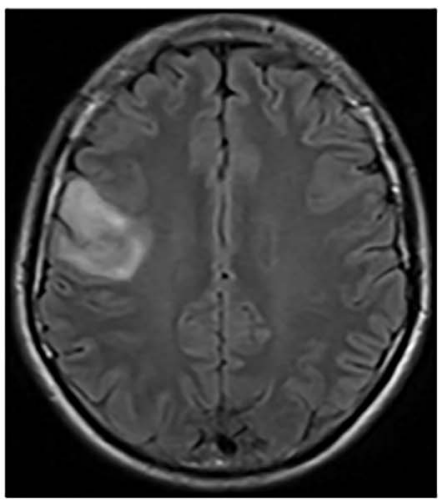

class 2: tumors invading 1 gyrus with extension to white matter and/or adjacent gyrus

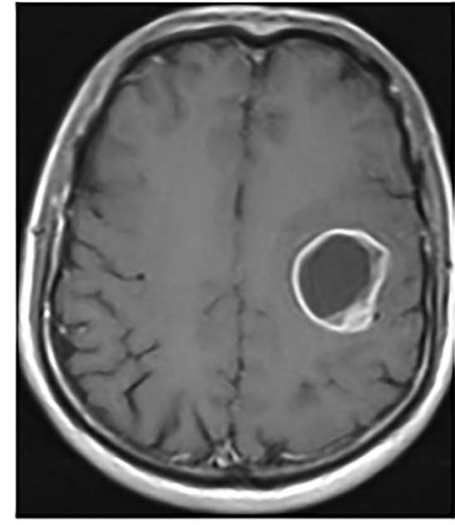

class 4: tumors primarily located in the white matter under eloquent gyri

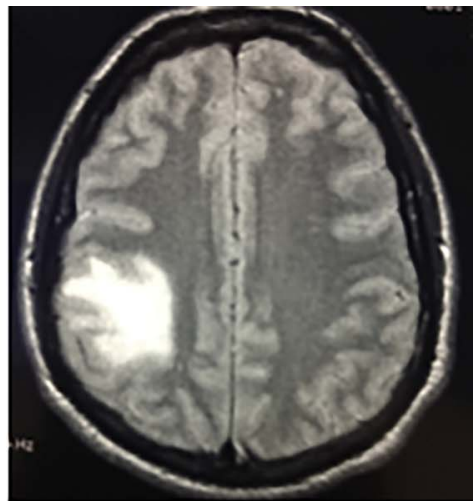

class 3: tumors infiltrating up to 3 gyri and extending toward the long range white matter tracts

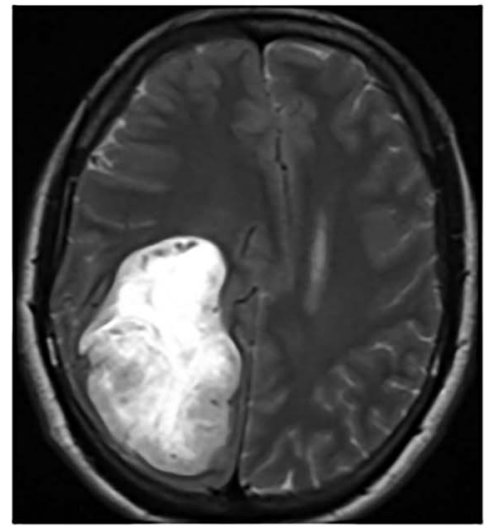

class 5: lobar tumors.

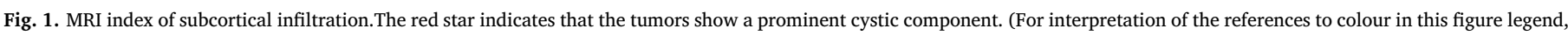
the reader is referred to the web version of this article.)

Table 1

Neuroradiological and clinical factors which are considered to calculate the prognostic score (PS).

\begin{tabular}{llll}
\hline+ & sharp margins & yes [1] & no [0] \\
+ & cyst & yes [1] & no [0] \\
+ & seizures at onset & yes [1] & no [0] \\
- & MRI index $>2$ & yes [1] & no [0] \\
- & volume $>80 \mathrm{~cm} 3$ & yes [1] & no [0] \\
- & presumptive higher grade of malignancy & yes [1] & no [0] \\
- & paresis/dysesthesia & yes [1] & no [0] \\
\hline
\end{tabular}

identified factors with the best predictive power to create an in-house prognostic score (PS). We decided to apply this PS to only sensorimotor area gliomas, rather than language area gliomas, as these tumors are associated with less variable cortico-subcortical structures (i.e. primary motor cortex, premotor cortex, primary sensory areas, and corticospinal tract).

Here, we describe the results of our prospective study on this novel clinico-radiological PS that can help define the surgical strategy for patients with sensorimotor area gliomas.

\section{Patients and methods}

Form December 2012 to April 2016, we analyzed from a prospectively collected database of patients diagnosed with brain gliomas of the sensorimotor area. The inclusion criteria were as follows: the presence of suspected glioma infiltrating or in close vicinity to the precentral or postcentral gyri and cortico-spinal tract; availability of preoperative and follow-up clinical and neuroradiological data; and intraoperative confirmation of eloquence through intraoperative monitoring or cortico-subcortical electrical stimulation. The last criterion was vital to enhance the reliability of the PS. The positions of the sensory and motor areas were determined anatomically (axial T1 and T2) or through functional magnetic resonance imaging and diffusion tensor imaging (in cases where the precentral and postcentral gyri and CST were severely compressed or dislocated). When feasible, we follow a criteria of proposing awake surgery in patients with sensorimotor area tumors in order to obtain more detailed mapping, particularly in cases with important subcortical infiltration. However, intraoperative monitoring (IOM) in an asleep patient was chosen when the patient was unsuitable for awake surgery, and the tumor volume and subcortical infiltration were limited. Exclusion criteria were as follow: age under 18; patients with relapse/progression of a previously operated tumor.

The preoperative data used to calculate the PS included clinical and neuroradiological parameters. Clinical parameters were: the presence of seizures at onset and presence of paresis/dysesthesias. These deficits were considered regardless they responded or not to steroid administration. Neuroradiological parameters were: 1. morphology of the 
Table 2

Clinical, radiological and histological patients' data (mRS, modified Rankin Score; HGG, high grade glioma).

\begin{tabular}{|c|c|c|c|}
\hline mean age (range) & $48(20-72)$ & & \\
\hline female/male ratio & $17 / 27$ & & \\
\hline awake surgery/IOM & $34 / 10$ & & \\
\hline \multicolumn{4}{|l|}{ clinical presentation } \\
\hline seizures at onset & $\begin{array}{l}\text { paresis/dysesthesia at } \\
\text { onset }\end{array}$ & & \\
\hline $28(63.6 \%)$ & $19(43.1 \%)$ & & \\
\hline \multicolumn{4}{|l|}{ neuroimaging } \\
\hline $\begin{array}{l}\text { mean preoperative } \\
\text { volume } \mathrm{cm} 3 \\
\text { (range) }\end{array}$ & sharp margins & $\begin{array}{l}\text { presence of } \\
\text { cysts }\end{array}$ & $\begin{array}{l}\text { MRI } \\
\text { group }>2\end{array}$ \\
\hline $90.4(12.5-213.2)$ & $29(65.9 \%)$ & $12(27.2 \%)$ & $26(59 \%)$ \\
\hline \multicolumn{4}{|l|}{ histology } \\
\hline $\begin{array}{l}\text { presumed HGG (WHO } \\
\text { III-IV) }\end{array}$ & confirmed HGG & & \\
\hline $25(56.8 \%)$ & $29(65.9 \%)$ & & \\
\hline \multicolumn{4}{|l|}{ functional status } \\
\hline & Mean (SD) & $\begin{array}{l}\text { Range } \\
\text { [min-max] }\end{array}$ & \\
\hline PS & $4.36(1.37)$ & $1-7$ & \\
\hline mRS pre & $0.98(1.07)$ & $0-4$ & \\
\hline mRS post & $2.00(1.35)$ & $0-4$ & \\
\hline mRS follow-up & $0.95(1.10)$ & $0-4$ & \\
\hline postoperative & postoperative & & \\
\hline immediate & definitive & & \\
\hline deterioration & deterioration & & \\
\hline $28(63.6 \%)$ & $5(11.3 \%)$ & & \\
\hline \multicolumn{4}{|l|}{ surgical results } \\
\hline total resection & subtotal resection & $\begin{array}{l}\text { partial } \\
\text { resection }\end{array}$ & \\
\hline $26(59 \%)$ & $17(38.6 \%)$ & $1(2.2 \%)$ & \\
\hline
\end{tabular}

\section{Table 3}

Crosstabs and Fisher's exact test for outcomes proxies and prognostic index. EOR $=$ Total resection if 1 ; $m R S=$ modified Ranking Score improved or unvaried if 1 ; Outcome $=$ Good $(E O R=1$ and $\mathrm{mRS}=1)$, Mixed $($ EOR or $\mathrm{mRS}=1)$ or Bad Outcome $(\mathrm{EOR}$ and $\mathrm{mRS}=0) ; \mathrm{PS}=$ Prognostic Score; Fet = Fisher's exact test, in parentheses the number of cells. In the columns PS ranged from 1 to 7 , in the rows are shown the values of the outcomes proxies, so for example in the PS $=2$ column there were 2 patients with Bad Outcome and 0 patients with Mixed or Good Outcome.

\begin{tabular}{lrrrllll}
\hline EOR/PS & $\mathbf{1}$ & $\mathbf{2}$ & $\mathbf{3}$ & $\mathbf{4}$ & $\mathbf{5}$ & $\mathbf{6}$ & $\mathbf{7}$ \\
$\mathbf{0}$ & 1 & 2 & 8 & 5 & 2 & 0 & 0 \\
$\mathbf{1}$ & 0 & 0 & 1 & 7 & 8 & 8 & 2 \\
Fet $(7 \times 2)=20.79, \mathrm{p}<$ & 0.001 & & & & & \\
$\mathrm{mRS} / \mathrm{PS}$ & $\mathbf{1}$ & $\mathbf{2}$ & $\mathbf{3}$ & $\mathbf{4}$ & $\mathbf{5}$ & $\mathbf{6}$ & $\mathbf{7}$ \\
$\mathbf{0}$ & 0 & 2 & 3 & 2 & 0 & 0 & 0 \\
$\mathbf{1}$ & 1 & 0 & 6 & 10 & 10 & 8 & 2 \\
Fet $(7 \times 2)=12.06, \mathrm{p}=$ & 0.017 & & & & & \\
Outcome/PS & $\mathbf{1}$ & $\mathbf{2}$ & $\mathbf{3}$ & $\mathbf{4}$ & $\mathbf{5}$ & $\mathbf{6}$ & $\mathbf{7}$ \\
$\mathbf{0}$ & 0 & 2 & 3 & 2 & 0 & 0 & 0 \\
$\mathbf{1}$ & 1 & 0 & 5 & 3 & 2 & 0 & 0 \\
$\mathbf{2}$ & 0 & 0 & 1 & 7 & 8 & 8 & 2
\end{tabular}

Fet $(7 \times 3)=25.16, \mathrm{p}=0.001$

\section{Table 4}

Aggregate percent of the PS and outcome proxies. EOR $=$ Total resection if 1 ; $\mathrm{mRS}=$ modified Ranking Score improved or unvaried if 1 ; Outcome $=$ Good $(\mathrm{EOR}=1$ and $\mathrm{mRS}=1$ ), Mixed (EOR or $\mathrm{mRS}=1$ ) or Bad Outcome (EOR and $\mathrm{mRS}=0$ ); PS $=$ Prognostic Score.

\begin{tabular}{llll}
\hline PS & EOR & mRS & Total Outcome \\
\hline $1-3$ & $8 \%$ positive & $50 \%$ positive & $58 \%$ success $(50 \%$ mixed $8 \%$ positive $)$ \\
4 & $58 \%$ positive & $83 \%$ positive & $83 \%$ success $(25 \%$ mixed $58 \%$ positive) \\
5 & $80 \%$ positive & $100 \%$ positive & $100 \%$ success $(20 \%$ mixed $80 \%$ positive) \\
$6-7$ & $100 \%$ positive & & $100 \%$ success $(100 \%$ positive) \\
\hline
\end{tabular}

tumor margins (sharp or diffuse); 2. presence of cysts (large cystic borders surrounding the tumor); 3 . tumor volume (in $\mathrm{cm}^{3}$; tumor's volume threshold was fixed at $80 \mathrm{~cm}^{3}$ ). Volume's calculation was performed by approximation to the volume of a sphere or ellipsoid on gadolinium-enhanced MRI or on T2 FLAIR for non-enhancing tumors; 4. degree of subcortical white matter infiltration based on our previously described MRI index of infiltration (Fig. 1). Briefly, the visual anatomical limit on MRI to define the infiltration of subcortical connections was the end of the sulcus. The MRI patterns of invasion of the subcortical white matter were classified into 5 groups: (1) tumors invading and confined to only 1 gyrus; (2) tumors invading 1 gyrus with extension to white matter and/or adjacent gyrus; (3) tumors infiltrating up to 3 gyri and extending toward the long range white matter tracts; (4) tumors primarily located in the white matter below eloquent gyri; and (5) lobar tumors presumed high tumor grade based on contrast uptake. All the neuroradiological features were assessed by at least one neuroimaging expert. Further details regarding the determination of these prognosticators have been described previously [20].

The end-points included the EOR and the postoperative neurological status, expressed as the modified Rankin Score (mRS), at 1 and 6 months. The EOR was defined as gross total resection (GTR) in cases where $\geq 95 \%$ of tumor volume was resected, subtotal resection (STR) in cases where $85-95 \%$ of tumor volume was resected, and partial resection (PR) in cases where $<85 \%$ of tumor volume was resected.

Once the clinical and neuroradiological parameters were recorded, a value of 0 or 1 was assigned based on the presence or absence of that specific factor, as shown in Table 1 . These values were then added or subtracted based on the following formula:

PS $=5+$ Margins + Cysts + Seizure - Paresis - MRI index $>2$ Volume $>80 \mathrm{~cm}^{3}$ - Contrast enhancement. The resulting values range between 1 and 8 , wherein a higher value is indicative of a better outcome.

\subsection{Intraoperative protocol}

Awake cortical and subcortical mapping is performed through a bipolar fork measuring $6 \mathrm{~mm}$ in distance between the electrodes which delivers a non-deleterious, biphasic square-wave current in 4-s trains at $60 \mathrm{~Hz}$. Stimulation began at $1 \mathrm{~mA}$ and increases by $0.50 \mathrm{~mA}$ until generation of contralateral side movement or a paraesthesia occur. Every positive site is restimulated to confirm reproducibility of stimuli. For subcortical tumors, we test motor or sensory sites throughout the subcortical resection, stopping whenever anomalies appear. Motor responses following cortical and subcortical stimulation are checked via both direct observation of the patient and electromyography (EMG).

In asleep patients, intraoperative identification of the central sulcus and the central region was made using a combination of somatosensory evoked potential phase reversal and direct monopolar anodal highfrequency electrical stimulation of the cortex.

\subsection{Statistical analysis}

Statistical analyses and descriptive statistics were performed using IBM SPSS Statistics 20.0.0 software (SPSS ${ }^{\circ}$, IBM ${ }^{\circ}$, https://www.ibm. com/analytics/us/en/technology/spss).

We computed the descriptive statistics based on the demographic and clinical data of the sample. We used 3 proxies to evaluate the positive/negative outcome: EOR (total or subtotal); 6-month follow-up $\mathrm{mRS}$ (worsened or equal/improved compared to the preoperative mRS); and a combination of EOR and mRS (0: subtotal resection and worsened mRS; 1: total resection or better mRS; or 2: total resection and better $\mathrm{mRS}$ ).

We also assessed the predictive power of the PS using various analyses. Crosstabs and Fisher's exact test (Fet) analyses were used to evaluate the possible predictive parameters, as well as the classification of positive or negative outcomes for the chosen proxies; the significance 
Table 5

Functional differences in subgroups with high or low prognostic index. PS $=$ Prognostic Score: $\mathrm{mRS}=$ modified Rankin Score. Mean (SD), in bold $\mathrm{p}<0.05$.

\begin{tabular}{llll}
\hline Score & PS $<4$ & PS $\geq \mathbf{4}$ & $\mathbf{p}$ \\
$\mathrm{N}$ & 12 & 32 & - \\
mRS pre & $1.2(1.2)$ & $0.9(1.0)$ & 0.305 \\
mRS post & $2.7(1.6)$ & $1.7(1.2)$ & 0.043 \\
mRS follow up & $1.9(1.3)$ & $0.6(0.8)$ & $<\mathbf{0 . 0 0 1}$ \\
mRS post-pre & $-1.4(1.1)$ & $-0.9(1.2)$ & 0.189 \\
mRS follow up-pre & $-0.7(1.2)$ & $0.3(0.8)$ & $\mathbf{0 . 0 1 9}$ \\
\hline
\end{tabular}

Table 6

Comparison of binary classification for different indicators. Numbers of correctly classified patients $(\%) . \mathrm{EOR}=$ Total resection if $1 ; \mathrm{mRS}=$ modified Ranking Score improved or unvaried if 1 ; PS = Prognostic Score; HGG = presumptive higher grade of malignancy.

\begin{tabular}{lll}
\hline Cut-off & EOR & mRS \\
\hline PS [4 or more] & $39(89 \%)$ & $35(80 \%)$ \\
Margins [y] & $35(80 \%)$ & $24(55 \%)$ \\
Cystic [y] & $24(55 \%)$ & $19(43 \%)$ \\
Volume $>80 \mathrm{~cm}^{3}$ [y] & $17(39 \%)$ & $22(50 \%)$ \\
HGG [y] & $25(57 \%)$ & $28(64 \%)$ \\
Seizure [y] & $30(68 \%)$ & $29(66 \%)$ \\
Paresis [y] & $19(43 \%)$ & $20(45 \%)$ \\
MRI $>2[y]$ & $36(82 \%)$ & $25(57 \%)$ \\
\hline
\end{tabular}

threshold was set at $\mathrm{p}<0.05$.

Using 2-sample independent $t$-tests, we assessed the mRS at different time points (pre, post, and at 6 months) for 2 subgroups from the total sample based on a cut-off PS value of 4 (for further details refer to supplemental material).

\section{Results}

We enrolled a total of 48 patients with tumors in sensorimotor areas who were undergoing intraoperative mapping or monitoring. Of these patients, 4 were excluded as the tumor did not exhibit infiltration of the cortical nor subcortical sensorimotor regions intraoperatively. Table 2 describes the characteristics of the patients.

The PS was found to be associated with certain proxies, as determined by Fet (Table 3) and descriptive statistics (Table 4). For EOR, a PS value of $\geq 5$ was predictive of successful outcome, a value of 4 indicated an uncertain outcome, and a value of $\leq 3$ predicted a worse outcome. If only patients with a mRS on follow-up were considered, those with a PS of 4 exhibited worse or improved mRS values, those with a PS $\geq 5$ exhibited improved mRS values, and those with a PS $\leq 3$ exhibited worsened mRS values.

In the PS subgroups (Table 5), only the follow-up duration indicated a significant difference, including greater mRS values in those with PS $<4$ and a mean increase in the scores compared to those before surgery. In fact, the PS outperformed the single indicators (both EOR

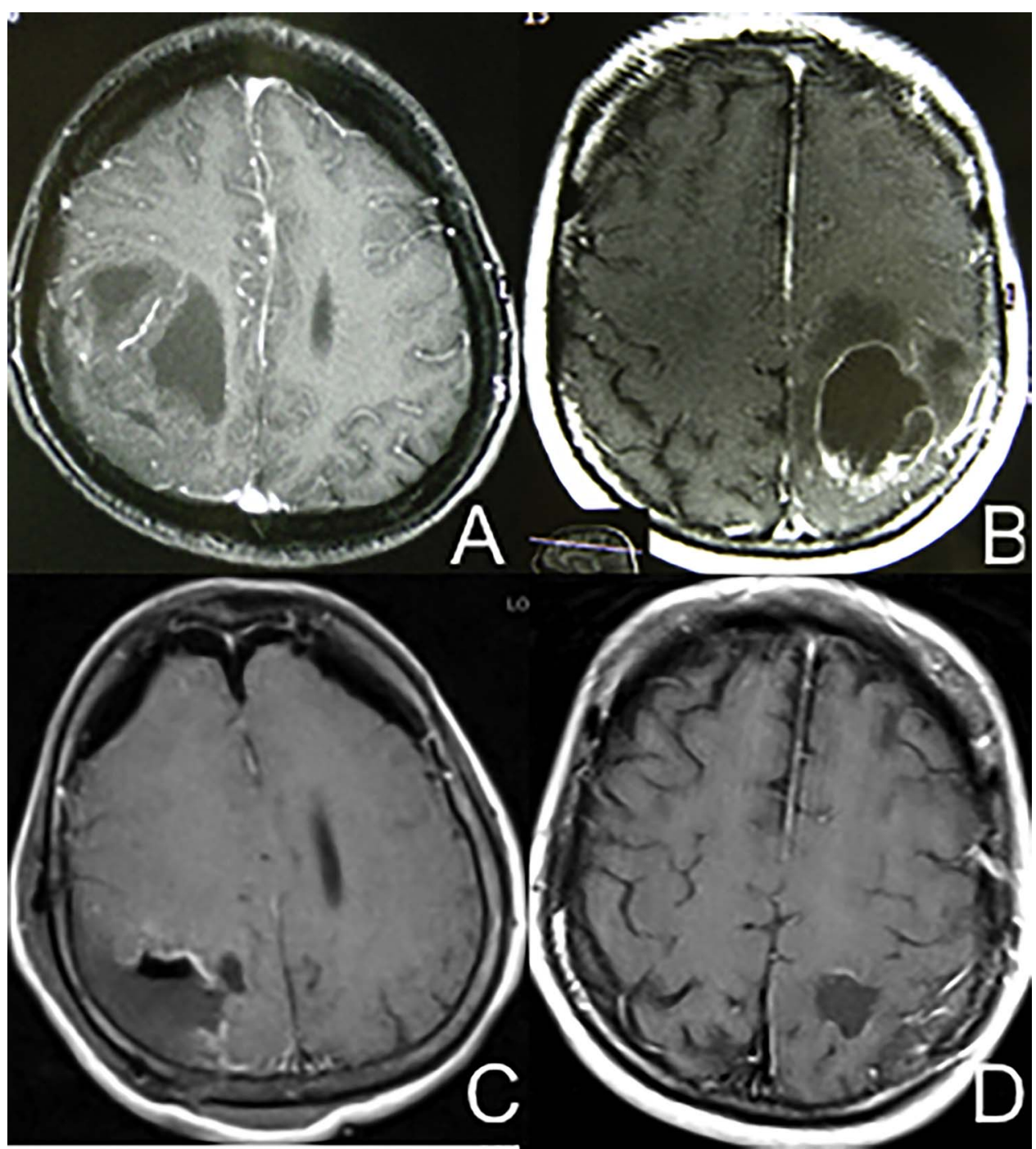

Fig. 2. Upper pictures present two tumors in similar location but different volume. Both had round margins and cystic component in contact with the CST. Seizures were the only complaint for both patients (patient B had also a very slight sensory syndrome). Tumors had characteristics of high grade gliomas.The PS for these patients was equally 5 although tumor in A was almost double in volume. In the postoperative period both patient had optimal evolution and complete recovery to fully functioning life. Both had complete resection as showed in $\mathrm{C}$ and D. Tumor A was an anaplastic oligodendroglioma and B was glioblastoma. 


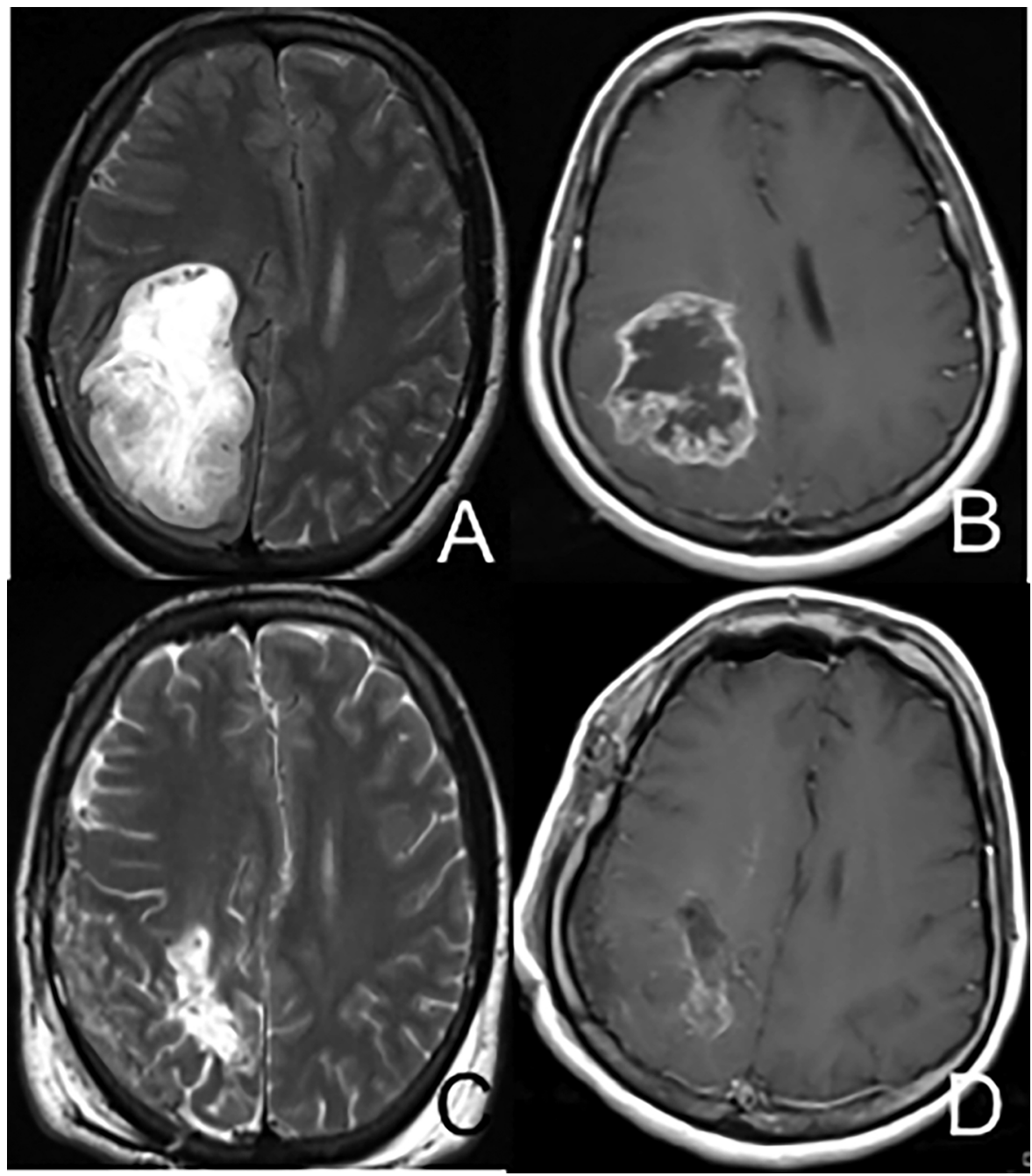

Fig. 3. The figure A shows a voluminous tumor extending into the whole parietal lobe toward the CST. The tumor has sharp margins and no contrast enhancement. Patient suffered from long-lasting seizures and slight left sensory syndrome. The PS for this patient is 4 meaning a degree of uncertainty about the functional outcome and EOR. In B a tumor in similar location but showing characteristics of higher grade of malignancy with ill-defined margins; the volume was lower than the former. The patient presented with severe paresis. In this case the PS was 2. C and D report the postoperative MRI showing complete resection in the first patient and subtotal resection in the second patient. This patient experienced also a worsening of functional status which did not recovered.

and mRS). In the Table 6 a comparison of binary classification for different indicators with the numbers of correctly classified patients.

\section{Discussion}

When counseling a patient with a tumor located in highly functional regions of the brain, it is ideal to use methods that accurately predict the EOR and functional outcome; in such cases, treatment aims to achieve a low incidence of postoperative permanent neurological deficits, as these would markedly affect the global outcome of patients by delaying adjuvant therapies and eventually reducing survival [2-4]. Such methods would help surgeons choose the best treatment strategy for specific patients, particularly when considering challenging operations, such as awake surgery and direct brain mapping. Surgeons typically consider several factors before choosing a surgical technique, such as the neurological status, symptoms at onset, tumor volume, proximity to eloquent regions, mass effect, and presumed malignancy. Although these are important factors to consider, only limited information is available on their role and contribution to the outcome (Fig. 2).

Tumor location is classically a predominant factor influencing the EOR and functional outcome. Historically, hemispheric gliomas are classified as far, near, or within eloquent areas, which are related to increasing risk of postoperative functional deterioration $[7,12,21,22-24]$. However, this categorization method is quite reductive and unreliable due to the wide interindividual functional variability, and even with the help of modern neuroradiologic advancements such as functional MRI and diffusion tensor imaging, it is difficult to predict the EOR and functional outcome [24-27]. Accordingly, intraoperative mapping techniques are becoming more commonly used, as they enable point-by-point mapping of the cortex and subcortical tracts with high reliability and reproducibility, as confirmed by large outcome studies $[24,28,29]$. Our PS is supported by systematic intraoperative confirmation through direct stimulation of the cortical and subcortical pathways. This point should be emphasized, because as intraoperative confirmation is not possible, the eloquent location of a tumor remains putative. Thus, a preoperative PS based only on functional or anatomical neuroimaging data may have very low reliability due to the interindividual variability and plasticity of the functional areas.

Tumor volume is another critical factor that influences surgery. However, in a previous study, we found that, although tumor volume is a determinant for safe surgery, it cannot be solely considered as a prognosticator and should instead be combined with other patientspecific factors (subcortical infiltration pattern, grade of malignancies, presence of preoperative deficits) to generate better predictions. This concept is evident in the present study, where apparently similar tumors behave in markedly different manners (Fig. 3). A reason for this phenomenon may involve the microscopic relationship between the pathologic tissue and the apparently healthy brain (i.e. tumor's sharp rounded margins can displace subcortical long tracts better then tumors with diffuse margins). Moreover, the growth rate can affect the brain capacity to reshape and adapt. This point is strictly related to the biology of the tumor (high or low grade) and influences the eventual 
appearance of focal deficits at onset. Furthermore, the central role of white matter infiltration on EOR and functional outcome has only recently been recognized $[30,31]$. Interestingly, this was not strictly related to the volume of the tumor $[20,30,31]$.

Based on our results, it appears that PS has good predictive value in discerning between good and bad outcomes, which may be due to the fact that we restricted analysis to only glial and sensorimotor area tumors. We aimed to create a PS that was applicable to a well-defined (both clinically and radiologically) type of patient and tumor. As mentioned previously, tumors located in the so-called "language" areas should be considered differently, due to the large networking structure of those areas and the wide spectrum of clinical presentations. The presence of a "gray zone"-i.e., a PS of 4-is important as it reflects a common condition where it is difficult to predict reliable outcomes.

The current PS can be applied in cases where the surgeon believes the tumor is located in proximity of the sensorimotor area. In fact, given that the PS value functions well in our series of cases with intraoperatively demonstrated sensorimotor area infiltration, it will also be suitable for tumors located in proximity to the sensorimotor areas.

It has to be emphasized that such a PS can help in formulating the potential risk in operating on sensory-motor located gliomas but it cannot indicate which kind of surgical techniques to be used (intraoperative monitoring, direct mapping etc.).

One limitation of the present study is that the number of patients is low and were strictly selected. However, this selection strategy was vital to obtain a homogeneous cohort of patients and yield more coherent results. Another possible bias may be related to the fact that all patients were initially intended to undergo surgery. Moreover, the grouping of HHG and LLG cases together may confound the results. In fact, we aimed to demonstrate the manner in which the growth rate and biology of tumors influence the interaction between the tumor and brain through different mechanisms (infiltration, dislocation, and plasticity), which finally affect the EOR and functional outcome. We foresee a new analysis in which HGG and LGG are analyzed separately.

\section{Conclusions and perspectives}

Even though it did not include a large sample, this prospective series clearly showed that the PS can be easily used in the clinical setting to help predicting the functional outcome and EOR. However, we believe that this PS should be integrated with other parameters (including fMRI, DTI, and transcranial magnetic stimulation) to generate a multifaceted score. Moreover, novel MRI spectroscopy software can also yield vital information on new biomarkers that can help define tumor malignancy and indications of the final outcome [32,33].

\section{Acknowledgments}

None.

\section{Appendix A. Supplementary data}

Supplementary data associated with this article can be found, in the online version, at http://dx.doi.org/10.1016/j.clineuro.2017.11.009.

\section{References}

[1] P.C. De Witt Hamer, S.G. Robles, A.H. Zwinderman, H. Duffau, M.S. Berger, Impact of intraoperative stimulation brain mapping on glioma surgery outcome: a metaanalysis, J. Clin. Oncol. 30 (2012) 2559-2565.

[2] S. Gulati, A.S. Jakola, U.S. Nerland, C. Weber, O. Solheim, The risk of getting worse: surgically acquired deficits, perioperative complications, and functional outcomes after primary resection of glioblastoma, World Neurosurg. 76 (2012) 572-579.

[3] A.S. Jakola, S. Gulati, C. Weber, G. Unsgård, O. Solheim, Postoperative deterioration in health related quality of life as predictor for survival in patients with glioblastoma: a prospective study, PLoS One 6 (2012) e28592.

[4] M.J. McGirt, D. Mukherjee, K.L. Chaichana, K.D. Than, J.D. Weingart, A. QuinonesHinojosa, Association of surgically acquired motor and language deficits on overall survival after resection of glioblastoma multiforme, Neurosurgery 65 (3) (2009) 463-469.

[5] F. Pignatti, M. van den Bent, D. Curran, C. Debruyne, R. Sylvester, et al., Prognostic factors for survival in adult patients with cerebral low-grade glioma. European Organization for Research and Treatment of Cancer Brain Tumor Cooperative Group, J. Clin. Oncol. 1520 (8) (2002) 2076-2084.

[6] E.R. Laws, I.F. Parney, W. Huang, F. Anderson, A.M. Morris, A. Asher, et al., Survival following surgery and prognostic factors for recently diagnosed malignant glioma: data from the Glioma Outcomes Project. Glioma Outcomes Investigators, Neurosurgery 99 (3) (2003) 467-473.

[7] T.Y. Jung, S. Jung, J.H. Moon, I.Y. Kim, K.S. Moon, et al., Early prognostic factors related to progression and malignant transformation of low-grade gliomas, Clin. Neurol. Neurosurg. 113 (9) (2011) 752-757.

[8] S.A. Yeh, J.T. Ho, C.C. Lui, Y.J. Huang, C.Y. Hsiung, et al., Treatment outcomes and prognostic factors in patients with supratentorial low-grade gliomas, Br. J. Radiol. 78 (927) (2005) 230-235.

[9] T. Gorlia, M.J. van den Bent, M.E. Hegi, R.O. Mirimanoff, M. Weller, et al., Nomograms for predicting survival of patients with newly diagnosed glioblastoma: prognostic factor analysis of EORTC and NCIC trial 26981-22981/CE. 3, Lancet Oncol. 9 (2008) 29-38.

[10] J.F. Mineo, A. Bordron, M. Baroncini, C. Ramirez, C.A. Maurage, et al., Prognosis factors of survival time in patients with glioblastoma multiforme: a multivariate analysis of 340 patients, Acta Neurochir. (Wien) 149 (2007) 245-253.

[11] U. Pichlmeier, A. Bink, G. Schackert, W. Stummer, ALA Glioma Study Group. Resection and survival in glioblastoma multiforme: an RTOG recursive partitioning analysis of ALA study patients, Neuro Oncol. 10 (6) (2008) 1025-1034.

[12] M. Lacroix, D. Abi-Said, D.R. Fourney, Z.L. Gokaslan, W. Shi, et al., A multivariate analysis of 416 patients with glioblastoma multiforme: prognosis, extent of resection, and survival, J. Neurosurg. 95 (2) (2001) 190-198.

[13] M.J. McGirt, K.L. Chaichana, M. Gathinji, F.J. Attenello, K. Than, et al., Independent association of extent of resection with survival in patients with malignant brain astrocytoma, J. Neurosurg. 110 (1) (2009) 156-162.

[14] H. Duffau, Intraoperative cortico-subcortical stimulations in surgery of low-grade gliomas, Expert Rev. Neurother. 5 (2005) 473-485.

[15] G.E. Keles, D.A. Lundin, K.R. Lamborn, E.F. Chang, G. Ojemann, et al., Intraoperative subcortical stimulation mapping for hemispherical perirolandic gliomas located within or adjacent to the descending motor pathways: evaluation of morbidity and assessment of functional outcome in 294 patients, J. Neurosurg. 100 (2004) 369-375.

[16] H. Duffau, P. Gatignol, E. Mandonnet, L. Capelle, L. Taillandier, Intraoperative subcortical stimulation mapping of language pathways in a consecutive series of 115 patients with Grade II glioma in the left dominant hemisphere, J. Neurosurg. 109 (2008) 461-471.

[17] M.S. Berger, A.V. Deliganis, J. Dobbins, G.E. Keles, The effect of extent of resection on recurrence in patients with low grade cerebral hemisphere gliomas, Cancer 74 (1994) 1784-1791.

[18] G. Spena, A. Nava, F. Cassini, A. Pepoli, M. Bruno, et al., Preoperative and intraoperative brain mapping for the resection of eloquent-area tumors. A prospective analysis of methodology, correlation, and usefulness based on clinical outcomes, Acta Neurochir. 152 (11) (2010) 1835-1846.

[19] R.L. Jensen, Predicting Outcomes After Glioma Surgery: Model Behavior, World Neurosurg. 84 (October (4)) (2015) 894-896.

[20] G. Spena, F. D'Agata, P.P. Panciani, Buglione di Monale M, Fontanella MM Supratentorial gliomas in eloquent areas: which parameters can predict functionaloutcome and extent of resection? PLoS One 8 (December (12)) (2013) e80916.

[21] S.S. Kim, I.E. McCutcheon, D. Suki, J.S. Weinberg, R. Sawaya, F.F. Lang, D. Ferson, A.B. Heimberger, F. DeMonte, S.S. Prabhu, Awake craniotomy for brain tumors near eloquent cortex: correlation of intraoperative cortical mapping with neurological outcomes in 309 consecutive patients, Neurosurgery 64 (May (5)) (2009) 836-845.

[22] E.F. Chang, J.S. Smith, S.M. Chang, K.R. Lamborn, M.D. Prados, N. Butowski, N.M. Barbaro, A.T. Parsa, M.S. Berger, McDermott MM Preoperative prognostic classification system for hemispheric low-grade gliomas in adults, J. Neurosurg. 109 (5) (2008 Nov) 817-824.

[23] M. Brell, J. Ibáñez, L. Caral, E. Ferrer, Factors influencing surgical complications of intra-axial brain tumours, Acta Neurochir. 142 (7) (2000) 739-750.

[24] F.E. Roux, K. Boulanouar, J.P. Ranjeva, C. Manelfe, M. Tremoulet, J. Sabatier, I. Berry, Cortical intraoperative stimulation in brain tumors as a tool to evaluate spatial data from motor functional MRI, Invest. Radiol. 34 (1999) 225-229.

[25] S. Lehéricy, H. Duffau, P. Cornu, L. Capelle, B. Pidoux, Carpentier Correspondence between functional magnetic resonance imaging somatotopy and individual brain anatomy of the central region: comparison with intraoperative stimulation in patients with brain tumors, J. Neurosurg. 92 (4) (2000) 589-598.

[26] A. Bizzi, V. Blasi, A. Falini, P. Ferroli, M. Cadioli, U. Danesi, D. Aquino, C. Marras, D. Caldiroli, G. Broggi, Presurgical functional MR imaging of language and motor functions: validation with intraoperative electrocortical mapping, Radiology 248 (2) (2008) 579-589.

[27] N. Petrovich, A.I. Holodny, V. Tabar, D.D. Correa, J. Hirsch, P.H. Gutin, C.W. Brennan, Discordance between functional magnetic resonance imaging during silent speech tasks and intraoperative speech arrest, J. Neurosurg. 103 (2005) 267-274

[28] A. De Benedictis, S. Moritz-Gasser, H. Duffau, Awake mapping optimizes the extent of resection for low-grade gliomas in eloquent areas, Neurosurgery 66 (6) (2010) 1074-1084.

[29] O. Sacko, V. Lauwers-Cances, D. Brauge, M. Sesay, A. Brenner, F.E. Roux, Awake craniotomy vs surgery under general anesthesia for resection of supratentorial lesions, Neurosurgery 68 (5) (2011) 1192-1198. 
[30] A. Castellano, L. Bello, C. Michelozzi, M. Gallucci, E. Fava, A. Iadanza, M. Riva, G. Casaceli, Falini A Role of diffusion tensor magnetic resonance tractography in predicting the extent of resection in glioma surgery, Neuro Oncol. 14 (2) (2012) 192-202.

[31] I.F. Talos, K.H. Zou, L. Ohno-Machado, J.G. Bhagwat, R. Kikinis, P.M. Black, Jolesz FA Supratentorial low-grade glioma resectability: statistical predictive analysis based on anatomic MR features and tumor characteristics, Radiology 239 (May (2)) (2006) 506-513.

[32] C. Choi, J.M. Raisanen, S.K. Ganji, S. Zhang, S.S. McNeil, Z. An, A. Madan, K.J. Hatanpaa, V. Vemireddy, C.A. Sheppard, D. Oliver, K.M. Hulsey, V. Tiwari,
T. Mashimo, J. Battiste, S. Barnett, C.J. Madden, T.R. Patel, E. Pan, C.R. Malloy, B.E. Mickey, R.M. Bachoo, E.A. Maher, Prospective longitudinal analysis of 2-hydroxyglutarate magnetic resonance spectroscopy identifies broad clinical utility for the management of patients with IDH-mutant glioma, J. Clin. Oncol. 34 (33) (2016) 4030-4039.

[33] A. Tietze, C. Choi, B. Mickey, E.A. Maher, B. Parm Ulhøi, R. Sangill, Y. LassenRamshad, S. Lukacova, L. Østergaard, G. von Oettingen, Noninvasive assessment of isocitrate dehydrogenase mutation status in cerebral gliomas by magnetic resonance spectroscopy in a clinical setting, J. Neurosurg. (March (3)) (2017) 1-8. 